\title{
The Application of Hyaluronic Acid-Based Hydrogels in Bone and Cartilage Tissue Engineering
}

\author{
Hongru Li $\mathbb{D}^{D}$, Zhiping Qi $\mathbb{D}^{\mathbb{D}}$, Shuang Zheng $\mathbb{D}^{\mathbb{D}}$, Yuxin Chang $\mathbb{D}^{D}$, Weijian Kong, Chuan Fu $(\mathbb{D}$, \\ Ziyuan Yu $\mathbb{D}^{D}$, Xiaoyu Yang $\mathbb{D}$, and Su Pan $\mathbb{D}$ \\ Department of Orthopedic Surgery, The Second Hospital of Jilin University, Ziqiang Street No. 218, Changchun TX 130041, China \\ Correspondence should be addressed to Xiaoyu Yang; yangxiaoyu88@sina.com and Su Pan; pansu78@sina.com
}

Received 20 April 2019; Revised 28 October 2019; Accepted 26 November 2019; Published 20 December 2019

Academic Editor: Antonio Gloria

Copyright (C) 2019 Hongru Li et al. This is an open access article distributed under the Creative Commons Attribution License, which permits unrestricted use, distribution, and reproduction in any medium, provided the original work is properly cited.

\begin{abstract}
At present, the healing of osteopathy, especially the healing of cartilage, has been proven to be difficult. Commonly used treatment methods are autogenous bone grafts and allogeneic bone grafts, but grafts cannot fully meet the clinical treatment requirements due to problems related to the source, price, immunity, and other concerns. Thus, the combination of biomaterials and tissue engineering technology has become a new direction in research. Among studies on tissue engineering bone and cartilage materials, hydrogels that show biological activity, absorbability after degradability, plasticity, and easy preparation have become the focus. Hydrogels are used as extracellular matrix mimics. Although various materials are able to form hydrogels, hyaluronic acid and its derivatives are prominently used. Hyaluronic acid hydrogels have many advantages, such as promoting cell adhesion and proliferation and wound healing. They also demonstrate sufficient biological activity for stimulating a microenvironment for cell survival. However, their disadvantages require further modification and include a poor degradation rate and insufficient mechanical performance. In this paper, hyaluronic acid-based hydrogels, their modifications, applications, and mechanisms, as well as new techniques for processing hyaluronic acid hydrogels in bone and cartilage tissue engineering, are briefly reviewed, and their future prospects and directions for future work are discussed.
\end{abstract}

\section{Introduction}

Bone is a basic body structure that constitutes parts of the motor system and supports routine human activities. In bone diseases, bone defects appear as the major clinical manifestation. Generally, external interventions are required when bone defects occur because irreversible damage to the self-healing capabilities has already occurred in most bone defect areas and because bone defects lack blood vessels [1]. Cartilage also does not contain blood vessels, nerves, or lymph, and the growth and proliferation of cartilage cells is slow; thus, cartilage healing is also a challenge [2] in regenerative medicine. Furthermore, problems with autografts and allografts include the source and price as well as immunity and ethics concerns [3]. Currently, bone and cartilage tissue engineering materials consist of stem cells and biological materials; thus, more research is needed $[4,5]$. Hydrogels, which are three-dimensional hydrophilic polymer chain systems, contain the same water content as soft tissue, and their composition, structure, and dimensions have been proven to be suitable for tissue engineering research [6]. Hydrogel materials are divided into natural polymers and synthetic polymers. Natural polymers include hyaluronic acid (HA), fibrin, chitosan, alginate, and pullulan [7]. They possess unique biological activities and osteoconductivity but are often lacking in mechanical strength and immunogenicity. Synthetic polymers are under development and include polyethylene oxide (PEO), polyvinyl alcohol (PVA), polyacrylic acid, and polyacrylic-fumaricethylene glycol copolymer [8]. The biocompatibility of synthetic polymer hydrogels needs improvement. There have been several in-depth studies of the natural polymer HA [9-13]. As a constituent of the natural cytoplasmic matrix, favorable biological activity of HA and its ability to communicate with cells are undisputed. Though appropriate modifications have been applied to the physicochemical 
properties of HA hydrogels, desired constructions with better properties are still needed. In this paper, we review hydrogels composed of HA and their derivates in bone and cartilage tissue engineering, providing a reference for the future development of better hydrogels based on HA and its derivates.

1.1. Bone and Cartilage Injury. Bone defects can be caused by various pathological processes, including trauma, cancer, congenital deformities, and surgical reconstruction. Bone healing is complex because multiple reactions such as inflammation, hematoma organization, cell proliferation, and calcification usually appear in injury areas. Bone loss, infection, insufficient blood supply, and soft tissue injury all influence bone healing. Additionally, the self-healing capabilities of bone are limited by the cell source and complexities of osteogenesis [14]. Therefore, external intervention is needed. Presently, application of the "gold standard," namely, autologous bone grafts, is restricted by inadequate or inappropriate bone stock, particularly for the treatment of extensive bone defects $[15,16]$. Currently, the critical dimension necessary for bone self-healing is only $2.5 \mathrm{~cm}$ [17].

Once bone disease involves the joints, cartilage injury inevitably occurs. Cartilage does not contain blood vessels, nerves, or lymph, and it is maintained and nourished by chondrocytes and plasmids in its extracellular matrix (ECM) [18]. Cartilage ECM is composed of a collagen network and proteoglycans; hyaluronic acid (HA) is a proteoglycan. Proteoglycans consist of a core protein with several glycosaminoglycan (GAG) chains. The mechanical properties of various types of cartilage differ in terms of the composition of collagen and GAG $[19,20]$. The cartilage ECM is only sustained by chondrocytes, which account for $1 \%$ to $5 \%$ of the total volume of cartilage. The low cell density of chondrocytes in cartilage and the lack of blood vessels cause low tissue regeneration capacity. Fibrous cartilage, which possesses higher tissue hardenability, is always generated during the healing process, resulting in long-term performance issues [21-23]. To increase the joint function, the formation of natural joint or hyaline cartilage should be promoted instead of fibrous cartilage $[23,24]$. Disadvantages (i.e., the lack of cartilage cell sources, long harvest times, difficulties with fixing, periosteum hypertrophy and ablation, and poor effects in elderly patients [25-27]) remain in autogenous chondrocyte grafts, which have been used for many years. Therefore, investigations into tissue engineering technology, including stem cells and biological materials, are urgently needed $[27,28]$.

1.2. Bone and Cartilage Tissue Engineering. Bone and cartilage tissue engineering involves the use of cultures and in vitro amplification of autologous high-concentration osteoblasts, bone marrow stroma stem cells, or chondrocytes, which are then implanted into a natural or synthetic cell scaffold or ECM that shows good biocompatibility (BC) and absorbability after degradation. The cells grow in this precast biomaterial three-dimensional scaffold because it is capable of providing nourishment, gas exchange, and waste removal.
This cell hybrid material is then implanted into the bone defect area. Combined with growth factors and drugs, the seeded bone cells demonstrate continuous proliferation and differentiation, thus repairing the bone tissue defect while exhibiting gradual degradation of the biological materials (Figure 1) [29].

Three aspects critical to bone tissue engineering (BTE) are osteoconductivity, osteoinductivity, and osseointegration [30], which are the required properties for biological scaffolds. The ideal scaffold materials for bone or cartilage tissue engineering include materials with a natural ecology, BC, the capability to seed osteoprogenitor cells (with the priority being mesenchymal stem cells (MSCs)), differentiation and proliferation capabilities, a natural ECM that can promote cell growth, morphogenesis signals, and adequate nutrition. The above conditions can guarantee the success of the healing phase [31, 32]. As a representative material, hydrogels are being studied in-depth and are expected to industrialize the production of BTE or CTE.

\section{Use of Hydrogels in BTE and CTE}

Hydrogels are three-dimensional solids that consist of hydrophilic polymer chains held together by cross-links. Hydrogels are a promising material in bone and cartilage tissue engineering due to unique advantages including adjustable physicochemical properties, biological nature, BC, versatility, and high similarity to the natural ECM. The requirements for hydrogel use in BTE and CTE are widely believed to include the following:

(1) Maintaining the phenotype of the osteoblasts or chondrocytes

(2) Rebuilding the interface of bone and cartilage and lessening the generation of fibrous cartilage

(3) Mimicking the natural ECM to create an ecological microenvironment

(4) Providing a sufficient vascularization or secretory matrix to sustain nutrition and metabolism

(5) Displaying a suitable degradation rate and no biological hazard after degradation

(6) Possessing the ability to deliver growth factors and drugs $[6,15,27,33-36]$

At present, the polymers used for hydrogel generation are divided into natural polymers and synthetic polymers. Natural polymers include HA, chitosan, fibrin, gelatin, alginate, gellan gum, amylopectin, self-assembling peptides, and respective derivatives. Representative synthetic polymers are acrylate-fumarate copolymer (PPF), polylactic acid (PLA), polyglycolic acid (PGA), polycaprolactone (PCL), polyethylene glycol (PEG), poly- $N$-isopropylacrylamide (PNIPAAM), triblock segmented copolymers of polyethylene oxide-b-polypropylene oxide-polyethylene oxide (PEO/PPO/PEO), and amphiphilic triblock-segmented copolymers of polyethylene oxide- $\varepsilon$-caprolactone (PEO/PCL/ PEO). The above materials have been used in bone and cartilage tissue engineering [15, 27]. Although there are 


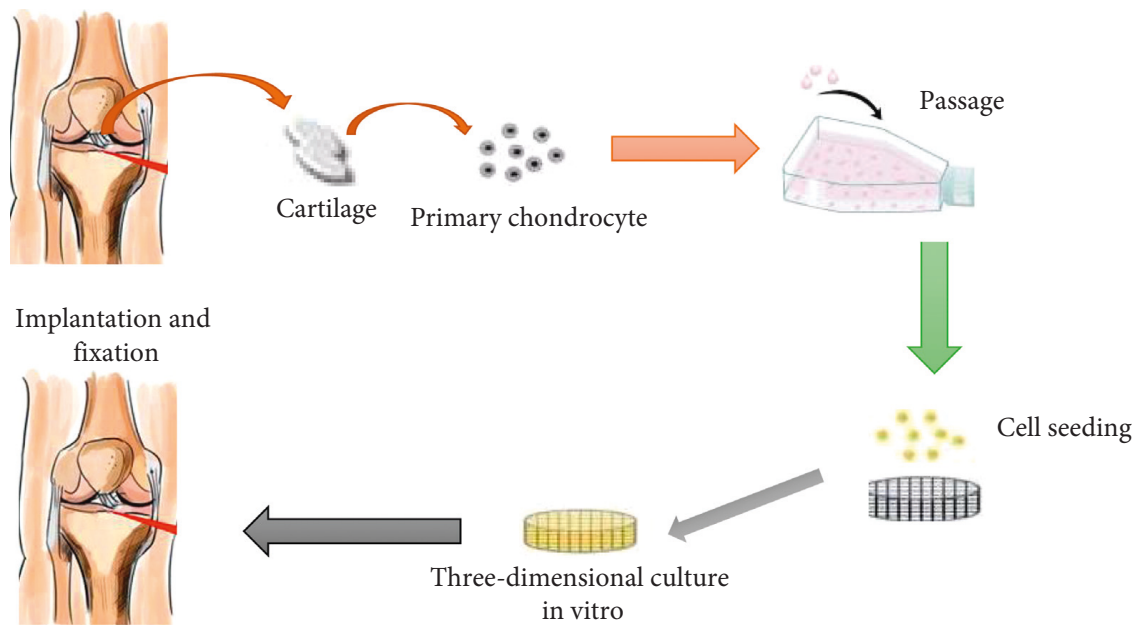

Figure 1: Schematic of cartilage tissue engineering.

many polymer choices, natural polymers with biological activity are preferred due to their unique characteristics, such as total BC, nonimmunogenicity, and nontoxicity. However, considering the instability, short half-life, and water content of biopolymers, natural polymers need to be modified before use. In particular, their water content, soft nature, and porous structure makes them able to mimic biological tissue and suitable for accommodating cells and encapsulating and releasing water-soluble compounds such as proteins in a controlled manner [37-39].

\section{HA-Based Hydrogels}

HA is a nonsulfated GAG consisting of repeating disaccharide units of glucuronic acid and $\mathrm{N}$-acetylglucosamine, which are widely distributed in cartilage [40, 41]. BTE and CTE have shown remarkable potential for HA-based hydrogels. The bioactivity of HA in cell signaling, wound repair, morphogenesis, and matrix composition depends on its structure and biological properties [42, 43]. Reviews assessing the promotion of the chondrocyte metabolism by HA [44] show that it can increase the synthetic amount of chondroitin-6-sulfate, collagen II, GAG, hydroxyproline, and DNA [45]. When delivering MSCs, HA-based hydrogels induce greater morphological differentiation of stem cells and help rebuild cartilage tissue. Meanwhile, it also provides lubrication and buffering effects for recovering the viscosity and elasticity of synovia $[44,46]$. More importantly, through minimally invasive surgery, injectable HA-based hydrogels are capable of completely repairing small-scale bone and cartilage defects of any size. However, HA has been primarily used in early CTE efforts due to its special influence on cartilage tissue and poor mechanical properties.

HA plays a significant role in promoting cell migration and proliferation. However, its use in vivo is limited by the endogenous degradation of hyaluronidase, reactive oxygen, and active nitrogen. Interactions between receptors, namely, CD44, hyaluronic acid-mediated motion receptor (RHAMM), and intercellular adhesion molecule-1 (ICAM1), and HA, have been identified in early studies. Further studies have been carried out to determine HA's effects on cell formation, wound repair, inflammation, and metastasis. For example, when modifying $\mathrm{HA}$ to synthesize HA macromolecule monomers, the interaction between CD44 and HA can be changed. This change depends on the degree of modification, but the exact mechanism requires further study. HA conjugate delivery nanoplatforms for the targeted treatment of CD44-positive metastatic tumors have also emerged. Recent research has further explored the interaction between HA and cell surface receptors, i.e., CD44, RHAMM, and ICAM-1 [47], which confirmed that these receptors influence cellular activities such as cell formation, wound repair, inflammation, and migration [48-51]. Due to the development of material manufacturing technologies, including various cross-linking methods, biological molecular binding [52], and 3D bioprinting, HA-based hydrogels have been increasingly used in both BTE and CTE applications.

\section{Modification of HA-Based Hydrogels for Application in BTE and CTE}

Due to disadvantages such as a hydrophilic nature and lack of mechanical integrity, HA requires chemical modification and cross-linking to alter it for use in BTE and CTE applications [53-55]. The most commonly used covalent modification sites of HA are the hydroxyl group and the carboxylic acid group (Figure 2). The hydroxyl group can be modified by an ester bond, whereas the carboxylic acid group is modified by hydrazide and then cross-linked with an ester bond. To improve its physicochemical properties, other functional groups can be added during modification. The partial deacetalization of HA via treatment with alkaline or acidic substances degrades HA into free amino groups that can be cross-linked by amides, imino groups, or secondary amine bonds. The bond energy of imino groups is recovered with amine bonds. Divinyl sulfone, glycol diglycidyl ether, carbodiimide, glutaric dialdehyde, sulfide, and polyfunctional epoxide can produce cross-linking reactions in acidic, neutral, or alkaline conditions. In addition, 


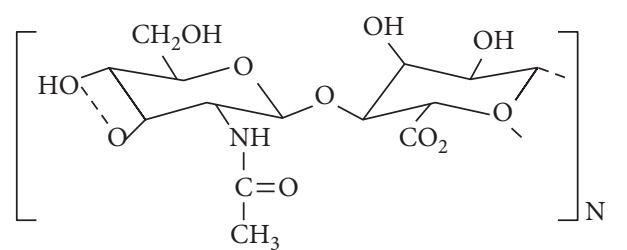

Figure 2: Structure of hyaluronic acid.

HA can undergo automatic cross-linking and photo-crosslinking in the presence of methacrylic acid ester. In addition to the abovementioned chemical modification methods, the combination of HA with other materials to form compound hydrogels has become a popular research topic.

\subsection{Chemical Modification and Application of HA Hydrogels}

4.1.1. Amidation Modification with Carbodiimide (EDC). The modification of HA reduces its degradation rate and solubility in water, guarantying its stability in BTE and CTE applications. The cross-linking of HA with carbodiimide promotes the formation of an ester bond between the hydroxyl group and the carboxyl group, which reacts with other functional groups (), increasing the porosity, rigidity, and antidegradation properties of the HA hydrogel scaffold. Therefore, carbodiimide has been used for the early modification of HA. La Gatta and colleagues [56] found that carbodiimide modification, HA scaffolds cross-linked with lysine (Lys), improves BC. Cross-linking of the amino bond improves the stability of HA scaffolds in the physiological environment, which is a major requirement for cartilage regeneration scaffolds. HA modification by carbodiimide can produce coupled reactions with mercaptamine to generate HA-hyaluronate thiol (HA-SH), which is then blended with a nanogel of another material to form a porous three-dimensional structure. Nanogel can be distributed uniformly. With high selectivity, speed, BC, and in situ polymerization, this newly biodegradable scaffold can be used as an injectable matrix coated with cells and proteins for tissue engineering and drug delivery applications [57].

Additionally, HA modified by both adipic dihydrazide $(\mathrm{ADH})$ and carbodiimide reacts with $N$-acryloxysuccinimide (NAS) to generate HA-acrylic acid (HA-AC), which can be used for gene delivery in vivo during the tissue regeneration process [58]. HA-AC cross-linking with 4-arm PEG thiol (PEG-SH4) has been used to create scaffolds of bone morphogenetic protein-2 (BMP-2) and bone MSCs (hMSCs) for the regeneration of rat skull defects [59]. To increase HA resistance, Pitarresi and colleagues [60] cross-linked HA modified by carbodiimide with $\alpha, \beta$-poly(N-2-ethoxy) (2-amino/ethyl/carbamate)-d, 1-asparaginate (PHEA-EDA) to form a proteoplast polymer gel membrane. The product features not only high hydratability and swelling resistance but also hydrolysis resistance and drug resistance against hyaluronidase. Photocurable hydrogels, which will be discussed later, also need early modification by carbodiimide.
4.1.2. Hydrazide Modification with Adipic Dihydrazide $(A D H)$. Hydrazide modification is used in the abovementioned preparation of HA-AC (Figure 3) [58, 59], which allows for further cross-linking to develop scaffolds for BTE and CTE applications. Scaffolds of porous HA with hydrazide modification have also been used to deliver BMP-2 for bone growth, with in vitro applications showing the continuous controlled release time of BMP-2 for up to a month. Histological analysis and staining after implantation of BMP-2 scaffolds into rats have revealed active bone regeneration and controlled BMP-2 release [61]. Further crosslinking with $\mathrm{ADH}$ derivatives can deliver macromolecule bifunctional reagents that can improve the swelling properties and drug resistance of HA. One example is methoxy PEG-propionaldehyde [62], which can be used for drug delivery after the formation of porous microstructural hydrogel membranes. $\mathrm{ADH}$ derivatives can also enhance cartilage differentiation by further binding with polypeptides to produce BMP-2 [63]. HA modified with hydrazide can be combined with aldehyde-modified HA through hydrazone cross-linking and be used to BMP-2, which strengthens the regeneration of cartilage tissue [64].

4.1.3. Aldehyde Modification. In glutaric dialdehyde- (GTA-) induced acid conditions, 2-(2-aminophenyl) acetaldehyde dimethyl acetal reacts with HA to form hemiacetal groups or ether bonds. Cross-linking neighboring chains of acetal or hemiacetal groups forms an insoluble gel. However, the complete removal of GTA must be guaranteed through a later process due to its toxicity. HA modification by GTA can be used to prepare compound hydrogels. For example, resorbent scaffolds made from HA modified by polyglycolic acid (PGA) and GTA are available for the meniscus repairs, and experiments have found that the expression of a specific marker is increased on the meniscus via HA-PGA scaffolds [65]. HA modification by 2-(2-aminophenyl) acetaldehyde dimethyl acetal has been verified to improve BMP-2 delivery. At the same time, aldehyde-modified hydrogels combined with acylhydrazide polyvinyl alcohol (PVA) can effectively deliver nontoxic siRNAs that can be applied to MSCs to block the inhibition of the clinically related gene PLEKHO1. This effect was demonstrated in siRNA release experiments and through the rheological properties of siRNA-encapsulated hydrogels [66].

4.1.4. Divinyl Sulfone Modification. Cross-linking divinyl sulfone (DVS) and the hydroxyl group of HA generates an ether bond. The rapid cross-linking of DVS and HA in alkaline conditions generates a hydrogel with strong mechanical properties, high BC, and high biological affinity, which can then be used in BTE and CTE applications $[67,68]$. For example, Xu and colleagues [69] developed fixed heparin (HP)-HA granule (HGP) through inverse emulsion polymerization using DVS as the cross-linking agent. This spherical carrier containing nanoscale micropores can be used for coating growth factors. HP specifically binds to BMP-2, and its release kinetics can be adjusted by regulating the granulometric composition. DVS cross-linked 


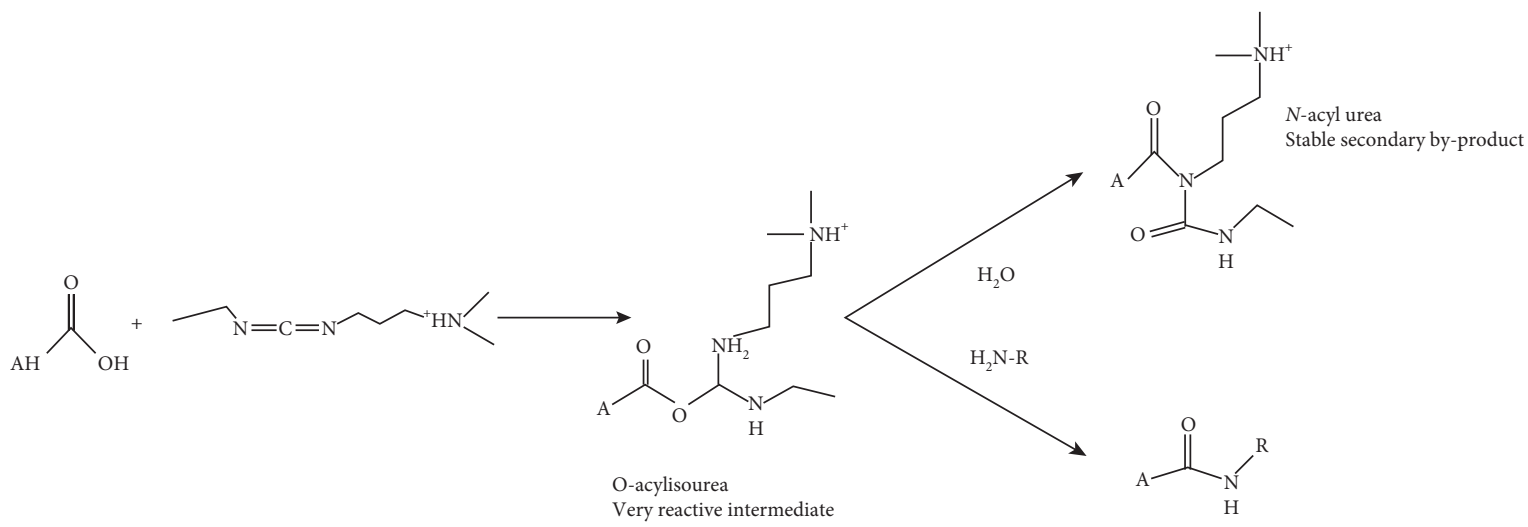

(a)

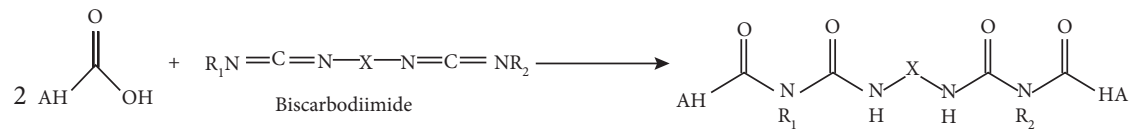

(b)

Figure 3: The HA amidation mechanism with EDC (a) and the cross-linking of HA with biscarbodiimides (b) [47].

hydrogels are also compatible with other materials such as carbon nanotubes [70].

4.1.5. Polyfunctional Epoxide Modification. The reaction of the epoxy group and hydroxide group of HA with hydroxide can generate an ester bond and ether bond. Kim and colleagues [71] used ethylene glycol diglycidyl ether to produce HA-collagen composite hydrogels for application in cartilage regeneration. An evaluation of its mechanical properties, degradation, and $\mathrm{BC}$ have concluded that this porous scaffold containing collagen can be effectively used in cartilage regeneration. Similarly, HA can react with 1,4-butanediol diglycidyl ether (BDDE) in alkaline conditions to generate a highly swelling hydrogel whose original shape remains unchanged [72].

Adding optical cross-linking to these hyperdistended hydrogels can increase the cross-linking density and limit swelling. After evaluation, they have been shown to be capable of being used for bone and cartilage tissue engineering scaffolds and molecular delivery.

4.1.6. Methacrylic Acid Modification. HA modified with methacrylic acid possess enhanced cellular affinity. Magalhaes and colleagues [73] found that chondrocytes adhere and present spherical morphology in the construction of HA hydrogels following methacrylic acid modification. Chondrocytes therein are able to proliferate and synthesize a hyaline matrix containing abundant glycosaminoglycans and collagen II. A common way to produce hydrogels is to use glycidyl methacrylate (GMA) to introduce photopolymerizable methacrylic acid ester on the molecule of HA to form GMHA [72], which can then be use to form HAbased hydrogels after photopolymerization. Irgacure 2959 is widely used as a photoinitiator in $N$-vinylpyrrolidone, which can not only maintain cell activity but also support the formation of hydrogels. Photo-cross-linking of HA methacrylic acid ester hydrogels (HAMAs) can also provide the $3 \mathrm{D}$ microenvironment that will allow MSCs to differentiate into the cartilage phenotype [74].

The reaction of $\mathrm{HA}$ and methacrylic acid generates MeHA containing methacrylic acid ester or binding functional groups containing acrylic ester (AHA), which can also permit photoinitiation cross-linking [75]. In the research of Erickson and colleagues [76], HA hydrogel construction based on hMSCs has been developed to repair articular cartilage defects through engineering approaches, and the highest modulus of compressibility has exceeded $1 \mathrm{MPa}$ during testing. 2-aminoethyl methacrylate (AEMA) can also cross-link as described above through amido bonds. As the substitution degree of AEMA has increased, research has shown [75] that the mechanical properties and swelling ratio of the HA hydrogels have improved. These two properties are keys for influencing the drug loading capacity and the proliferation, migration, and differentiation of cells. After methacrylic acid modification, the changes in the molecular weight of HA allow macromonomers such as polyethylene glycol diacrylate (PEGDM), in different concentrations, to mix in precursor solutions, forming a high cross-linking density network [77]. These networks have been used in animal experiments to maintain cell viability and generate new cartilage tissue.

Recently, there has been new research on improving compression stress, that is, employing the concept of double networks (DBs) to prepare high-intensive HA hydrogels using a two-step method [78]. First, HA is cross-linked to ethylene glycol diglycidyl ether (EGDE) in water; in the second step, the DB method is used to generate a DB hydrogel comprised of high-intensive HA/N, N-dimethylacrylamide (PDMA) containing $84-94 \%$ water. When compression stress increases to $19.4 \mathrm{MPa}$, these new hydrogels can be selected for BTE applications. 
In addition to photo-cross-linking, hydrogel granules of covalent integration (HA-c-HGP) [79] have been developed, which possess a higher tenacity and compression stress compared to traditional photo-cross-linked hydrogels. Cell adhesion peptides in connective tissue can be conjugated with acrylate or methacrylic acid ester functional groups on HA using the Michael addition reaction. A HA-based hydrogel has recently been developed by conjugating HA modified with acrylic acid to two different peptides. This HA-based hydrogel is sensitive to matrix metalloproteinase (MMP) and can be used as the carrier of cells and growth factors for bone formation [80, 81]. HA hydrogels modified with methacrylic acid and photo-cross-linking have also been used in 3D bioprinting [82], which will be introduced in Section 4.3.

4.1.7. Modification with Other Compounds. In addition to the abovementioned cross-linking modifications, other modifications adaptive to BTE and CTE have been studied. Lin and colleagues [83] prepared thiolated HA through disulfide bonds and cross-linking the former to PEGDM. When used to repair defects in the thighbone cartilage of the articularis patellar groove of rabbits, the results showed that bone and cartilage recovered well after 12 weeks. Cell adhesion peptide can also be conjugated to thiolated HA [84]. HA can also covalently cross-link tyramine in peroxide conditions, and the degree of hydrogel cross-linking can also adjust the matrix synthesis and cartilage histogeny. This injectable hydrogel possesses an adjustable three-dimensional microenvironment that is able to alter cell condensation during chondrogenesis and improve the histogeny of the overall cartilage tissue [85]. Kaderli and colleagues [86] found that HA-4-aminoresorcinol (HA$4 \mathrm{AR}$ ) hydrogels have protective effects on cartilage and can reduce the generation of synovium hypertrophy. Unterman and colleagues [87] used an HA-binding peptide (HABPep) to specifically bind HA to restrain HA-mediated delivery of hemameba. Hydrogel scaffolds of HABPep bound to polyethylene glycol diacrylate (PEGDA) are able to enhance cartilage repair in vivo, and this effect has been confirmed in a rat model of cartilage defects. Additionally, the hydroxyl group and carboxyl group (via the intermolecular bonds and intramolecular bonds that exist between them) can form self-cross-linking polymers, such as ACPTM, after esterification in vivo. These hydrogels have been used to reduce postoperative adhesions and have served as scaffolds for cell growth and repair in bone tissue defects [11].

4.2. Composite HA Hydrogels and Their Application. Composite HA hydrogels that consist of two or more natural/synthetic biopolymers incorporate the advantages of biopolymers, while eliminating some of the disadvantages through improved BC and biodegradability and adjustable mechanical strength. Therefore, research on composite HA hydrogels is appealing to the field of bone cartilage regeneration [88]. Using HAs modified by multiple methods and with other covalent binding materials, diversified composite HA hydrogels have been developed for use in BTE and CTE applications.

4.2.1. Binding to Collagen. Collagen is an abundant ingredient of bone, cartilage, and ECM [89]. It is believed that collagen is capable of facilitating the growth, proliferation, and conglutination of MSCs, as well as the formation and differentiation of cartilage [90]. Attempts to bind together collagen and HA have demonstrated better effects than using either one alone. For example, Liu [91] cross-linked activated formyl group-modified HA with collagen to form a new collagen-HA (COL/HA) matrix. Experimental results indicated that this matrix exhibited improved BC and osteoconduction in the skull of rats. Modified HA bound to collagen has also shown good performance [92] for inducing the osteogenic differentiation of hMSCs and assisting mineralization. It can also promote the gene expression of some bone components in order to promote bone formation. In CTE, collagen-HA scaffolds have shown high MSC infiltration, stimulating the motility of MSCs and their capacity for cartilage formation [71, 93]. To enhance mechanical strength, different concentrations of HA-dialdehyde (HAD), produced by periodate oxidation, combined with collagen to prepare collagen-HAD composite $(\mathrm{CH})$ gels have been verified as a bionic matrix that promotes cell migration and morphology changes [94].

4.2.2. Binding to Gelatin. Gelatin is the hydrolysate of collagen and is composed of many cell adhesion peptide (RGD) sequences and MMP target sequences that promote cell remodeling [41]. Therefore, it has received significant attention from the tissue engineering field. The combination of HA modified with mercaptan (Glycosil), gelatin modified with mercaptan (Gelin-S), and PEGDA has been used to form cell construction matrices that have shown good effects in CTE [46]. Chang and colleagues [95] prepared scaffolds using a trinal copolymer of porcine chondrocytes, gelatin/ chondroitin, and sulfate/HA to mimic the natural cartilage matrix to treat through thickness joint injuries in pigs with good efficacy. Similar experiments have also been conducted in rabbits [83]. We showed that the combination of photocross-linked GelHA hydrogels and ASCs enables ASC to differentiate into nucleus pulposus tissue and enhances the efficacy of ASCs in disc repair by activating the integrin $\alpha \mathrm{v} \beta 6$ 6-TGF-beta 1 pathway [96].

4.2.3. Binding to Fibrin. Fibrin containing RGDs can not only induce the aggregation of polymers but also have commendable BC [97]. Because they can be cross-linked or bound to reinforcing materials, HA bound to fibrin has been shown to increase mechanical strength and cell adhesion. Fibrin/HA-MA hydrogels [98]/HA-AC hydrogels [58]/PCL/ PLGA scaffolds coated with fibrin and HA admixtures [99] and PGA-HA scaffolds mixed with fibrin [65] have, respectively, been used in BTE and CTE applications (Table 1). 
Table 1: Binding to fibrin.

\begin{tabular}{|c|c|c|c|}
\hline Modification & Conformation & Biochemical function/applications & References \\
\hline MA & Fibrin/HA-MA hydrogel & $\begin{array}{l}\text { Increased mechanical strength, BMSC proliferation, } \\
\text { and chondrogenic potential in vitro }\end{array}$ & {$[98]$} \\
\hline EDC, ADH, AHA & Fibrin/HA-AC hydrogel & DNA delivery & {$[58]$} \\
\hline PCL/PLGA & $\begin{array}{l}\text { PCL/PLGA stent coated with a mixture of fibrin and } \\
\text { HA }\end{array}$ & $\begin{array}{l}\text { Used as a carrier for the delivery of BMP-2 and } \\
\text { ASCs, resulting in better bone formation and } \\
\text { mineralization }\end{array}$ & [99] \\
\hline PGA & Fibrin-stabilized 3D PGA-HA scaffold & $\begin{array}{l}\text { Cultures meniscus cells, promotes the re-expression } \\
\text { of meniscus-specific markers, and forms meniscus } \\
\text { matrix components }\end{array}$ & {$[65]$} \\
\hline
\end{tabular}

4.2.4. Binding to Alginate. Mahapatra and colleagues [100] prepared alginate-HA hydrogels mixed with collagen I. These Alg-HA-Col composite hydrogels demonstrated higher secretion of cartilage matrices, which can not only enhance mechanical strength and maintain biological stability and the biochemical environment but also contribute to the maintenance of the cell phenotype. Research on coating with both alginate microspheres containing TFGF$\beta 3$ and HA hydrogels containing human MSCs has demonstrated optimized MSC differentiation [101].

4.2.5. Binding to Chitosan. In addition to the abovementioned materials, chitosan is also a favorable biological binding material. Due to Schiff base reactions between the amidogen groups and aldehyde groups of polysaccharide derivatives, gelation does not require a cross-linking agent that shows toxicity on living cells. $\mathrm{N}$-succinyl-chitosan (SSC) and aldehyde-HA (A-HA) are used to prepare composite injectable hydrogels. Its hydraulic time, microstructure, surface morphology, equilibrium swelling, compression modulus, and degradation in vitro are suitable, and the effects of coating chondrocytes are obvious [102]. In experiments in rabbits, HA-acetylation chitosan hydrogels have surprisingly augmented subchondral bone remodeling and cartilage formation [86]. The porosity of lyophilized chitosan-HA-dialdehyde gels increases with the degree of oxidation, and chondrocytes coated in CHDA gel retain their viability and special phenotypic characteristics [102].

4.2.6. Binding to Other Materials. Chiari et al. [103] used new biological materials composed of $\mathrm{HA}$ and polycaprolactone as substitutions for sheep menisci. Solid-free form (SSF) preparations of polylactic-co-glycolic acidgrafted HA (HA-PLGA) polymers and polymers of ethylene glycol, and were then coated with BMP-2 for use on skull defects in rats. The effects were obvious [61]. Sheu [104] prepared oxydic HA/resveratrol (Oxi-HA/Res) hydrogels that were capable of $\mathrm{BC}$, allowed the formation of the ECM, and reduced the inflammations and injury induced by LPS. It has been verified that HA cross-linked to aggrecan and cartilage-linked proteins are better lubricants than Simplex HA coatings [105]. HA modified by adipic dihydrazide (ADH-HA) and HA modified by aldehyde (a-HA), which are enhanced by cellulose nanocrystals $(\alpha$-CNCs) with different contents of aldehyde modifications, have been used to form new injectable hydrogels. hASCs coated in HA-CNC hydrogels show diffusion and active proliferative activities in these hydrogels [106]. Hydroxyapatite nanoparticles blended with polyvinyl derivatives react with $\mathrm{HA}$ in situ to form a hydrogel, which then can be used to release BMP-2 for bone remodeling [68]. Pentosan polysulfate (a highly sulfating semisynthesis polysaccharide, PPS) shows chondrocyte induction potential. PEG/HA hydrogels have been used to deliver mesenchymal progenitor cells (MPCs), and covalently bound HA-PPS hydrogels can promote cartilage regeneration in treatments for multiple diseases, including intervertebral disc degeneration (IVD) [107]. Collagen/ chitosan/HA hydrogels cross-linked by genipin have excellent mechanical properties and extended degradation times. These hydrogels display a compact structure, which shows advantages in ossicle regeneration [108]. Heparin modified with mercaptan (Heprasil $^{\mathrm{TM}}$ ) and combined with HA modified by mercaptan hybridized PEDGA can adequately control the release of BMP-2 [109].

4.3. Other Modifications of HA Hydrogels. Though the abovementioned research on HA-based delivery of stem cells, growth factors, and drugs have shown promise and effects in bone regeneration, more work is needed to meet the functionality and complexity of mature tissue or organ construction in tissue engineering applications. With the continuous development of materials technology, research on the directional assembly of hydrogels is imperative. Electrostatic spinning, centrifugal casting, template leaching, and phase separation technologies are examples of newly emerged technologies [47]. These technologies have shown advantages for regulating and controlling the macroscopic pore size and shape but have limited benefits in terms of porosity and the exchange of biological information on a microscopic level. At present, the combination of 3D bioprinting and micropatterning has been the most studied [110].

With "biology ink" (cell aggregations or spheroids) and "biology paper" (scaffold materials), 3D bioprinting fabricates layers that are later stacked in the computer to create functional living tissues [111]. The technology two-photon polymerization (TPP) under femtosecond laser irradiation generates $3 \mathrm{D}$ submicron patterns on hydrogel scaffolds that have been gradually introduced into HA hydrogels and 3D bioprinting [112]. The methods for selecting biological ink and biological paper vary. 
Experiments have shown that the three kinds of composite material preparations of HA-g, HA-pHEA, and HAgelatin hydrogels have stable rheology, excellent $\mathrm{BC}$, and printability, showing their potential for use in tissue engineering in the future.

Methacrylic acidizing and photo-cross-linking are used to form hydrogels and scaffold materials that are then used for tubulose construction printing of HA-gelatin hydrogels [82]. New semi-interpenetrating polymer networks (semiIPNs) of dextran are derived from HA and ethoxyl metacrylic acid ester [113]. Furthermore, $24 \mathrm{~nm}$ gold nanoparticles (AuNPs) are dynamically cross-linked to HA modified with thiol and gelatin to form sECM hydrogels [114]. The abovementioned materials are conducive for $3 \mathrm{D}$ bioprinting.

\section{Conclusions and Prospects}

Despite the above advantages and modification methods, HA-based scaffolds still face challenges. HA-based scaffolds used in vivo can induce foreign body reactions. Various proteins can be absorbed onto the surfaces of implanted HAbased scaffolds and induce a series of degeneration effects. Moreover, scaffolds can induce an inflammatory reaction [115]. The generation and activation of hyaluronidase remains a problem to be solved for controlled release. In conclusion, with the support of a series of cross-linking technologies and materials technologies, HA hydrogels and their derivatives have shown great potential in BTE and CTE applications. It is believed that bionic matrices capable of highly mimicking the cellular environment will be important in the further refinement of bioreactors and scaffold construction.

\section{Conflicts of Interest}

There are no conflicts of interest to declare.

\section{Acknowledgments}

This study was supported by the National Natural Science Foundation of China (no. 31572217) and the Jilin Provincial Educational 135 Science and Technology Project (nos. JJKH20180224KJ and JJKH20180209KJ).

\section{References}

[1] T. A. Einhorn and L. C. Gerstenfeld, "Fracture healing: mechanisms and interventions," Nature Reviews Rheumatology, vol. 11, no. 1, pp. 45-54, 2014.

[2] B. Choi, S. Kim, B. Lin, B. M. Wu, and M. Lee, "Cartilaginous extracellular matrix-modified chitosan hydrogels for cartilage tissue engineering," ACS Applied Materials \& Interfaces, vol. 6, no. 22, pp. 20110-20121, 2014.

[3] B. Balakrishnan and R. Banerjee, "Biopolymer-based hydrogels for cartilage tissue engineering," Chemical Reviews, vol. 111, no. 8, pp. 4453-4474, 2011.

[4] J. Henkel, M. A. Woodruff, D. R. Epari et al., "Bone regeneration based on tissue engineering conceptions-a 21st century perspective," Bone Research, vol. 1, no. 3, pp. 216248, 2013.
[5] A. R. Amini, C. T. Laurencin, and S. P. Nukavarapu, "Bone tissue engineering: recent advances and challenges," Critical Reviews in Biomedical Engineering, vol. 40, no. 5, pp. 363408, 2012.

[6] B. V. Slaughter, S. S. Khurshid, O. Z. Fisher, A. Khademhosseini, and N. A. Peppas, "Hydrogels in regenerative medicine," Advanced Materials, vol. 21, no. 32-33, pp. 3307-3329, 2009.

[7] N. Annabi, A. Tamayol, J. A. Uquillas et al., "25th anniversary article: rational design and applications of hydrogels in regenerative medicine," Advanced Materials, vol. 26, no. 1, pp. 85-124, 2014.

[8] S. P. Borderud, Y. Li, J. E. Burkhalter, C. E. Sheffer, and J. S. Ostroff, "Electronic cigarette use among patients with cancer: characteristics of electronic cigarette users and their smoking cessation outcomes," Cancer, vol. 120, no. 22, pp. 3527-3535, 2014.

[9] B. P. Toole, "Hyaluronan: from extracellular glue to pericellular cue," Nature Reviews Cancer, vol. 4, no. 7, pp. 528-539, 2004.

[10] S. Trombino, C. Servidio, F. Curcio, and R. Cassano, "Strategies for hyaluronic acid-based hydrogel design in drug delivery," Pharmaceutics, vol. 11, no. 8, p. 407, 2019.

[11] Z. Li, C. Tang, D. Huang et al., "Sensitive hyaluronidase biosensor based on target-responsive hydrogel using electronic balance as readout," Analytical Chemistry, vol. 91, no. 18, pp. 11821-11826, 2019.

[12] G. D. Prestwich, "Engineering a clinically-useful matrix for cell therapy," Organogenesis, vol. 4, no. 1, pp. 42-47, 2008.

[13] Y.-J. Seong, G. Lin, B. J. Kim, H.-E. Kim, S. Kim, and S.-H. Jeong, "Hyaluronic acid-based hybrid hydrogel microspheres with enhanced structural stability and high injectability," ACS Omega, vol. 4, no. 9, pp. 13834-13844, 2019.

[14] R. Florencio-Silva, G. R. d. S. Sasso, E. Sasso-Cerri, M. J. Simões, and P. S. Cerri, "Biology of bone tissue: structure, function, and factors that influence bone cells," BioMed Research International, vol. 2015, Article ID 421746, 17 pages, 2015.

[15] A. R. Short, D. Koralla, A. Deshmukh et al., "Hydrogels that allow and facilitate bone repair, remodeling, and regeneration," Journal of Materials Chemistry B, vol. 3, no. 40, pp. 7818-7830, 2015.

[16] M. A. Talmaç, M. A. Görgel, M. Kanar, S. Erinç, A. H. Olçar, and H. M. Özdemir, "Anterior and posterior iliac crest bone graft donor site morbidities: a comparing retrospective study," Bakirkoy Tip Dergisi/Medical Journal of Bakirkoy, vol. 15, no. 3, pp. 244-249, 2019.

[17] E. H. Schemitsch, "Size matters," Journal of Orthopaedic Trauma, vol. 31, no. 5, pp. S20-S22, 2017.

[18] A.-M. Yousefi, M. E. Hoque, R. G. S. V. Prasad, and N. Uth, "Current strategies in multiphasic scaffold design for osteochondral tissue engineering: a review," Journal of Biomedical Materials Research Part A, vol. 103, no. 7, pp. 2460-2481, 2015.

[19] S. P. Nukavarapu and D. L. Dorcemus, "Osteochondral tissue engineering: current strategies and challenges," Biotechnology Advances, vol. 31, no. 5, pp. 706-721, 2013.

[20] A. J. Almarza and K. A. Athanasiou, "Design characteristics for the tissue engineering of cartilaginous tissues," Annals of Biomedical Engineering, vol. 32, no. 1, pp. 2-17, 2004.

[21] A. J. Sutherland, E. C. Beck, S. C. Dennis et al., "Decellularized cartilage may be a chondroinductive material for osteochondral tissue engineering," PLoS One, vol. 10, no. 5, Article ID e0121966, 2015. 
[22] Y. S. Kim, M. Majid, A. J. Melchiorri, and A. G. Mikos, "Applications of decellularized extracellular matrix in bone and cartilage tissue engineering," Bioengineering \& Translational Medicine, vol. 4, no. 1, pp. 83-95, 2019.

[23] H. Akkiraju and A. Nohe, "Role of chondrocytes in cartilage formation, progression of osteoarthritis and cartilage regeneration," Journal of Developmental Biology, vol. 3, no. 4, pp. 177-192, 2015.

[24] L. Baptista, G. Kronemberger, I. Côrtes et al., "Adult stem cells spheroids to optimize cell colonization in scaffolds for cartilage and bone tissue engineering," International Journal of Molecular Sciences, vol. 19, no. 5, p. 1285, 2018.

[25] R. S. Tuan, "A second-generation autologous chondrocyte implantation approach to the treatment of focal articular cartilage defects," Arthritis Research \& Therapy, vol. 9, no. 5, p. 109, 2007.

[26] P. Giannoni, A. Pagano, E. Maggi et al., "Autologous chondrocyte implantation (ACI) for aged patients: development of the proper cell expansion conditions for possible therapeutic applications," Osteoarthritis and Cartilage, vol. 13, no. 7, pp. 589-600, 2005.

[27] J. Yang, Y. S. Zhang, K. Yue, and A. Khademhosseini, "Cellladen hydrogels for osteochondral and cartilage tissue engineering," Acta Biomaterialia, vol. 57, pp. 1-25, 2017.

[28] W. J. McCarty, A. Luan, P. Sundaramurthy et al., "An arthroscopic device to assess articular cartilage defects and treatment with a hydrogel," Annals of Biomedical Engineering, vol. 39, no. 4, pp. 1306-1312, 2011.

[29] L. Roseti, V. Parisi, M. Petretta et al., "Scaffolds for bone tissue engineering: state of the art and new perspectives," Materials Science and Engineering: C, vol. 78, pp. 1246-1262, 2017.

[30] A. Malhotra and P. Habibovic, "Calcium phosphates and angiogenesis: implications and advances for bone regeneration," Trends in Biotechnology, vol. 34, no. 12, pp. 983-992, 2016.

[31] A. Dhawan, P. M. Kennedy, E. B. Rizk, and I. T. Ozbolat, "Three-dimensional bioprinting for bone and cartilage restoration in orthopaedic surgery," Journal of the American Academy of Orthopaedic Surgeons, vol. 27, no. 5, pp. e215e226, 2019.

[32] T. L. Arinzeh, "Mesenchymal stem cells for bone repair: preclinical studies and potential orthopedic applications," Foot and Ankle Clinics, vol. 10, no. 4, pp. 651-665, 2005.

[33] S.-H. Lee and H. Shin, "Matrices and scaffolds for delivery of bioactive molecules in bone and cartilage tissue engineering," Advanced Drug Delivery Reviews, vol. 59, no. 4-5, pp. 339-359, 2007.

[34] M. C. Catoira, L. Fusaro, D. Di Francesco, M. Ramella, and F. Boccafoschi, "Overview of natural hydrogels for regenerative medicine applications," Journal of Materials Science: Materials in Medicine, vol. 30, no. 10, p. 115, 2019.

[35] K. L. Spiller, S. A. Maher, and A. M. Lowman, "Hydrogels for the repair of articular cartilage defects," Tissue Engineering Part B: Reviews, vol. 17, no. 4, pp. 281-299, 2011.

[36] A. A. Amini and L. S. Nair, "Injectable hydrogels for bone and cartilage repair," Biomedical Materials, vol. 7, no. 2, p. $024105,2012$.

[37] K.-C. Chang, D.-J. Lin, Y.-R. Wu et al., "Characterization of genipin-crosslinked gelatin/hyaluronic acid-based hydrogel membranes and loaded with hinokitiol: in vitro evaluation of antibacterial activity and biocompatibility," Materials Science and Engineering: C, vol. 105, p. 110074, 2019.

[38] P. Makvandi, G. W. Ali, F. D. Sala, W. I. Abdel-Fattah, and A. Borzacchiello, "Biosynthesis and characterization of antibacterial thermosensitive hydrogels based on corn silk extract, hyaluronic acid and nanosilver for potential wound healing," Carbohydrate Polymers, vol. 223, p. 115023, 2019.

[39] G. Huang and J. Chen, "Preparation and applications of hyaluronic acid and its derivatives," International Journal of Biological Macromolecules, vol. 125, pp. 478-484, 2019.

[40] M. Sun, X. Sun, Z. Wang, S. Guo, G. Yu, and H. Yang, "Synthesis and properties of gelatin methacryloyl (GelMA) hydrogels and their recent applications in load-bearing tissue," Polymers, vol. 10, no. 11, p. 1290, 2018.

[41] K. Yue, G. Trujillo-de Santiago, M. M. Alvarez, A. Tamayol, N. Annabi, and A. Khademhosseini, "Synthesis, properties, and biomedical applications of gelatin methacryloyl (GelMA) hydrogels," Biomaterials, vol. 73, pp. 254-271, 2015.

[42] V. Nicola, S. Juergen, S. Robert, and S. Ladislav, "Role, metabolism, chemical modifications and applications of hyaluronan," Current Medicinal Chemistry, vol. 16, no. 14, pp. 1718-1745, 2009.

[43] K. Matsumoto, Y. Li, C. Jakuba et al., "Conditional inactivation of Has2 reveals a crucial role for hyaluronan in skeletal growth, patterning, chondrocyte maturation and joint formation in the developing limb," Development, vol. 136, no. 16, pp. 2825-2835, 2009.

[44] A. Stellavato, V. Vassallo, A. La Gatta et al., "Novel hybrid gels made of high and low molecular weight hyaluronic acid induce proliferation and reduce inflammation in an osteoarthritis in vitro model based on human synoviocytes and chondrocytes," BioMed Research International, vol. 2019, Article ID 4328219, 13 pages, 2019.

[45] J. A. Burdick and G. D. Prestwich, "Hyaluronic acid hydrogels for biomedical applications," Advanced Materials, vol. 23, no. 12, pp. H41-H56, 2011.

[46] W. S. Toh, E. H. Lee, X.-M. Guo et al., "Cartilage repair using hyaluronan hydrogel-encapsulated human embryonic stem cell-derived chondrogenic cells," Biomaterials, vol. 31, no. 27, pp. 6968-6980, 2010.

[47] C. A. Maxwell, J. McCarthy, and E. Turley, "Cell-surface and mitotic-spindle RHAMM: moonlighting or dual oncogenic functions?," Journal of Cell Science, vol. 121, no. 7, pp. 925-932, 2008.

[48] C. B. Knudson, "Hyaluronan and CD44: strategic players for cell-matrix interactions during chondrogenesis and matrix assembly," Birth Defects Research Part C: Embryo Today: Reviews, vol. 69, no. 2, pp. 174-196, 2003.

[49] B. Lu, Y. Li, Z. Wang et al., "A dual responsive hyaluronic acid graft poly(ionic liquid) block copolymer micelle for an efficient CD44-targeted antitumor drug delivery," New Journal of Chemistry, vol. 43, no. 31, pp. 12275-12282, 2019.

[50] K. J. Wolf and S. Kumar, "Hyaluronic acid: incorporating the bio into the material," ACS Biomaterials Science \& Engineering, vol. 5, no. 8, pp. 3753-3765, 2019.

[51] H. Fang, X. Zhao, X. Gu et al., "CD44-targeted multifunctional nanomedicines based on a single-component hyaluronic acid conjugate with all-natural precursors: construction and treatment of metastatic breast tumors in vivo," Biomacromolecules, 2019.

[52] S. Yamane, N. Iwasaki, T. Majima et al., "Feasibility of chitosan-based hyaluronic acid hybrid biomaterial for a novel scaffold in cartilage tissue engineering," Biomaterials, vol. 26, no. 6, pp. 611-619, 2005

[53] G. Prestwich and J.-w. Kuo, "Chemically-modified HA for therapy and regenerative medicine," Current Pharmaceutical Biotechnology, vol. 9, no. 4, pp. 242-245, 2008. 
[54] M. N. Collins and C. Birkinshaw, "Hyaluronic acid based scaffolds for tissue engineering-a review," Carbohydrate Polymers, vol. 92, no. 2, pp. 1262-1279, 2013.

[55] F. De Sarno, A. M. Ponsiglione, A. M. Grimaldi, P. A. Netti, and E. Torino, "Effect of crosslinking agent to design nanostructured hyaluronic acid-based hydrogels with improved relaxometric properties," Carbohydrate Polymers, vol. 222, p. 114991, 2019.

[56] A. La Gatta, G. Ricci, A. Stellavato et al., "Hyaluronan hydrogels with a low degree of modification as scaffolds for cartilage engineering," International Journal of Biological Macromolecules, vol. 103, pp. 978-989, 2017.

[57] S. A. Bencherif, N. R. Washburn, and K. Matyjaszewski, "Synthesis by AGET ATRP of degradable nanogel precursors for in situ formation of nanostructured hyaluronic acid hydrogel," Biomacromolecules, vol. 10, no. 9, pp. 2499-2507, 2009.

[58] Y. Lei, M. Rahim, Q. Ng, and T. Segura, "Hyaluronic acid and fibrin hydrogels with concentrated DNA/PEI polyplexes for local gene delivery," Journal of Controlled Release, vol. 153, no. 3, pp. 255-261, 2011.

[59] J. Kim, I. S. Kim, T. H. Cho et al., "Bone regeneration using hyaluronic acid-based hydrogel with bone morphogenic protein-2 and human mesenchymal stem cells," Biomaterials, vol. 28, no. 10, pp. 1830-1837, 2007.

[60] G. Pitarresi, F. S. Palumbo, R. Calabrese, E. F. Craparo, and G. Giammona, "Crosslinked hyaluronan with a protein-like polymer: novel bioresorbable films for biomedical applications," Journal of Biomedical Materials Research Part A, vol. 84A, no. 2, pp. 413-424, 2008.

[61] J. K. Park, J.-H. Shim, K. S. Kang et al., "Solid free-form fabrication of tissue-engineering scaffolds with a poly(lacticco-glycolic acid) grafted hyaluronic acid conjugate encapsulating an intact bone morphogenetic protein-2/poly(ethylene glycol) complex," Advanced Functional Materials, vol. 21, no. 15, pp. 2906-2912, 2011.

[62] Y. Luo, K. R. Kirker, and G. D. Prestwich, "Cross-linked hyaluronic acid hydrogel films: new biomaterials for drug delivery," Journal of Controlled Release, vol. 69, no. 1, pp. 169-184, 2000.

[63] A. K. Jha, W. Yang, C. B. Kirn-Safran, M. C. Farach-Carson, and X. Jia, "Perlecan domain I-conjugated, hyaluronic acidbased hydrogel particles for enhanced chondrogenic differentiation via BMP-2 release," Biomaterials, vol. 30, no. 36, pp. 6964-6975, 2009.

[64] E. Martínez-Sanz, D. A. Ossipov, J. Hilborn, S. Larsson, K. B. Jonsson, and O. P. Varghese, "Bone reservoir: injectable hyaluronic acid hydrogel for minimal invasive bone augmentation," Journal of Controlled Release, vol. 152, no. 2, pp. 232-240, 2011.

[65] U. Freymann, M. Endres, K. Neumann, H.-J. Scholman, L. Morawietz, and C. Kaps, "Expanded human meniscusderived cells in 3-D polymer-hyaluronan scaffolds for meniscus repair," Acta Biomaterialia, vol. 8, no. 2, pp. 677-685, 2012.

[66] M. Paidikondala, G. N. Nawale, and O. P. Varghese, "Insights into siRNA transfection in suspension: efficient gene silencing in human mesenchymal stem cells encapsulated in hyaluronic acid hydrogel," Biomacromolecules, vol. 20, no. 3, pp. 1317-1324, 2019.

[67] E. Martínez-Sanz, O. P. Varghese, M. Kisiel et al., "Minimally invasive mandibular bone augmentation using injectable hydrogels," Journal of Tissue Engineering and Regenerative Medicine, vol. 6, no. S3, pp. s15-s23, 2012.
[68] A.-C. Docherty-Skogh, K. Bergman, M. J. Waern et al., "Bone morphogenetic protein-2 delivered by hyaluronan-based hydrogel induces massive bone formation and healing of cranial defects in minipigs," Plastic and Reconstructive Surgery, vol. 125, no. 5, pp. 1383-1392, 2010.

[69] X. Xu, A. K. Jha, R. L. Duncan, and X. Jia, "Heparin-decorated, hyaluronic acid-based hydrogel particles for the controlled release of bone morphogenetic protein 2," Acta Biomaterialia, vol. 7, no. 8, pp. 3050-3059, 2011.

[70] S. Bhattacharyya, S. Guillot, H. Dabboue, J.-F. Tranchant, and J.-P. Salvetat, "Carbon nanotubes as structural nanofibers for hyaluronic acid hydrogel scaffolds," Biomacromolecules, vol. 9, no. 2, pp. 505-509, 2008.

[71] H. J. Kim, K. K. Kim, I. K. Park, B. S. Choi, J. H. Kim, and M. S. Kim, "Erratum to: hybrid scaffolds composed of hyaluronic acid and collagen for cartilage," Tissue Engineering and Regenerative Medicine, vol. 9, no. 4, p. 231, 2012.

[72] S. Zawko, S. Suri, Q. Truong, and C. Schmidt, "Photopatterned anisotropic swelling of dual-crosslinked hyaluronic acid hydrogels," Acta Biomaterialia, vol. 5, no. 1, pp. 14-22, 2009.

[73] J. Magalhães, A. Crawford, P. V. Hatton, F. J. Blanco, and J. S. Roman, "Poly(2-ethyl-(2-pyrrolidone) methacrylate) and hyaluronic acid-based hydrogels for the engineering of a cartilage-like tissue using bovine articular chondrocytes," Journal of Bioactive and Compatible Polymers, vol. 29, no. 6, pp. 545-559, 2014.

[74] C. Chung and J. A. Burdick, "Influence of three-dimensional hyaluronic acid microenvironments on mesenchymal stem cell chondrogenesis," Tissue Engineering Part A, vol. 15, no. 2, pp. 243-254, 2009.

[75] I. L. Kim, R. L. Mauck, and J. A. Burdick, "Hydrogel design for cartilage tissue engineering: a case study with hyaluronic acid," Biomaterials, vol. 32, no. 34, pp. 8771-8782, 2011.

[76] I. E. Erickson, S. R. Kestle, K. H. Zellars et al., "High mesenchymal stem cell seeding densities in hyaluronic acid hydrogels produce engineered cartilage with native tissue properties," Acta Biomaterialia, vol. 8, no. 8, pp. 3027-3034, 2012.

[77] J. A. Burdick, C. Chung, X. Jia, M. A. Randolph, and R. Langer, "Controlled degradation and mechanical behavior of photopolymerized hyaluronic acid networks," Biomacromolecules, vol. 6, no. 1, pp. 386-391, 2005.

[78] B. Tavsanli and O. Okay, "Preparation and fracture process of high strength hyaluronic acid hydrogels cross-linked by ethylene glycol diglycidyl ether," Reactive and Functional Polymers, vol. 109, pp. 42-51, 2016.

[79] A. K. Jha, M. S. Malik, M. C. Farach-Carson, R. L. Duncan, and X. Jia, "Hierarchically structured, hyaluronic acid-based hydrogel matrices via the covalent integration of microgels into macroscopic networks," Soft Matter, vol. 6, no. 20, pp. 5045-5055, 2010.

[80] J. Kim, I. S. Kim, T. H. Cho et al., "In vivo evaluation of MMP sensitive high-molecular weight HA-based hydrogels for bone tissue engineering," Journal of Biomedical Materials Research Part A, vol. 95A, no. 3, pp. 673-681, 2010.

[81] Y. Park, S. B. Yu, Y. I. Chung et al., "Bone regeneration with MMP sensitive hyaluronic acid-based hydrogel, rhBMP-2 and nanoparticles in rat calvarial critical size defect (CSD) model," Journal of the Korean Association of Oral and Maxillofacial Surgeons, vol. 35, pp. 137-145, 2009.

[82] A. Skardal, J. Zhang, L. McCoard, X. Xu, S. Oottamasathien, and G. D. Prestwich, "Photocrosslinkable hyaluronan-gelatin hydrogels for two-step bioprinting," Tissue Engineering Part A, vol. 16, no. 8, pp. 2675-2685, 2010. 
[83] Y. Liu, X. Z. Shu, and G. D. Prestwich, "Osteochondral defect repair with autologous bone marrow-derived mesenchymal stem cells in an injectable, in situ, cross-linked synthetic extracellular matrix," Tissue Engineering, vol. 12, no. 12, pp. 3405-3416, 2006.

[84] S. Khetan and J. A. Burdick, "Patterning network structure to spatially control cellular remodeling and stem cell fate within 3-dimensional hydrogels," Biomaterials, vol. 31, no. 32, pp. 8228-8234, 2010.

[85] W. S. Toh, T. C. Lim, M. Kurisawa, and M. Spector, "Modulation of mesenchymal stem cell chondrogenesis in a tunable hyaluronic acid hydrogel microenvironment," Biomaterials, vol. 33, no. 15, pp. 3835-3845, 2012.

[86] S. Kaderli, E. Viguier, D. Watrelot-Virieux et al., "Efficacy study of two novel hyaluronic acid-based formulations for viscosupplementation therapy in an early osteoarthrosic rabbit model," European Journal of Pharmaceutics and Biopharmaceutics, vol. 96, pp. 388-395, 2015.

[87] S. A. Unterman, M. Gibson, J. H. Lee et al., "Hyaluronic acidbinding scaffold for articular cartilage repair," Tissue Engineering Part A, vol. 18, no. 23-24, pp. 2497-2506, 2012.

[88] O. Jeznach, D. Kołbuk, and P. Sajkiewicz, "Injectable hydrogels and nanocomposite hydrogels for cartilage regeneration," Journal of Biomedical Materials Research Part A, vol. 106, no. 10, pp. 2762-2776, 2018.

[89] W. Zhao, X. Jin, Y. Cong, Y. Liu, and J. Fu, "Degradable natural polymer hydrogels for articular cartilage tissue engineering," Journal of Chemical Technology \& Biotechnology, vol. 88, no. 3, pp. 327-339, 2013.

[90] B. Marquass, J. S. Somerson, P. Hepp et al., "A novel MSCseeded triphasic construct for the repair of osteochondral defects," Journal of Orthopaedic Research, vol. 28, no. 12, pp. 1586-1599, 2010.

[91] L.-S. Liu, A. Y. Thompson, M. A. Heidaran, J. W. Poser, and R. C. Spiro, "An osteoconductive collagen/hyaluronate matrix for bone regeneration," Biomaterials, vol. 20, no. 12, pp. 1097-1108, 1999.

[92] U. Hempel, S. Möller, C. Noack et al., "Sulfated hyaluronan/ collagen I matrices enhance the osteogenic differentiation of human mesenchymal stromal cells in vitro even in the absence of dexamethasone," Acta Biomaterialia, vol. 8, no. 11, pp. 4064-4072, 2012.

[93] A. Matsiko, T. J. Levingstone, F. J. O’Brien, and J. P. Gleeson, "Addition of hyaluronic acid improves cellular infiltration and promotes early-stage chondrogenesis in a collagen-based scaffold for cartilage tissue engineering," Journal of the Mechanical Behavior of Biomedical Materials, vol. 11, pp. 41-52, 2012.

[94] R. Mohan, N. Mohan, and D. Vaikkath, "Hyaluronic acid dictates chondrocyte morphology and migration in composite gels," Tissue Engineering Part A, vol. 24, no. 19-20, pp. 1481-1491, 2018.

[95] C.-H. Chang, T.-F. Kuo, C.-C. Lin et al., "Tissue engineeringbased cartilage repair with allogenous chondrocytes and gelatin-chondroitin-hyaluronan tri-copolymer scaffold: a porcine model assessed at 18, 24, and 36 weeks," Biomaterials, vol. 27, no. 9, pp. 1876-1888, 2006.

[96] P. Chen, L. Ning, P. Qiu et al., "Photo-crosslinked gelatinhyaluronic acid methacrylate hydrogel-committed nucleus pulposus-like differentiation of adipose stromal cells for intervertebral disc repair," Journal of Tissue Engineering and Regenerative Medicine, vol. 13, no. 4, pp. 682-693, 2019.

[97] J. S. Park, H. N. Yang, D. G. Woo, S. Y. Jeon, and K.-H. Park, "Chondrogenesis of human mesenchymal stem cells in fibrin constructs evaluated in vitro and in nude mouse and rabbit defects models," Biomaterials, vol. 32, no. 6, pp. 1495-1507, 2011.

[98] T. N. Snyder, K. Madhavan, M. Intrator, R. C. Dregalla, and D. Park, "A fibrin/hyaluronic acid hydrogel for the delivery of mesenchymal stem cells and potential for articular cartilage repair," Journal of Biological Engineering, vol. 8, no. 1, p. 10, 2014.

[99] S.-W. Kang, J.-S. Kim, K.-S. Park et al., "Surface modification with fibrin/hyaluronic acid hydrogel on solid-free formbased scaffolds followed by BMP-2 loading to enhance bone regeneration," Bone, vol. 48, no. 2, pp. 298-306, 2011.

[100] C. Mahapatra, G.-Z. Jin, and H.-W. Kim, "Alginate-hyaluronic acid-collagen composite hydrogel favorable for the culture of chondrocytes and their phenotype maintenance," Tissue Engineering and Regenerative Medicine, vol. 13, no. 5, pp. 538-546, 2016.

[101] L. Bian, D. Y. Zhai, E. Tous, R. Rai, R. L. Mauck, and J. A. Burdick, "Enhanced MSC chondrogenesis following delivery of TGF- $\beta 3$ from alginate microspheres within hyaluronic acid hydrogels in vitro and in vivo," Biomaterials, vol. 32, no. 27, pp. 6425-6434, 2011.

[102] H. Tan, C. R. Chu, K. A. Payne, and K. G. Marra, "Injectable in situ forming biodegradable chitosan-hyaluronic acid based hydrogels for cartilage tissue engineering," Biomaterials, vol. 30, no. 13, pp. 2499-2506, 2009.

[103] C. Chiari, U. Koller, R. Dorotka et al., "A tissue engineering approach to meniscus regeneration in a sheep model," Osteoarthritis and Cartilage, vol. 14, no. 10, pp. 1056-1065, 2006.

[104] S.-Y. Sheu, W.-S. Chen, J.-S. Sun, F.-H. Lin, and T. Wu, "Biological characterization of oxidized hyaluronic acid/ resveratrol hydrogel for cartilage tissue engineering," Journal of Biomedical Materials Research Part A, vol. 101, no. 12, pp. 3457-3466, 2013.

[105] J. Seror, Y. Merkher, N. Kampf et al., "Articular cartilage proteoglycans as boundary lubricants: structure and frictional interaction of surface-attached hyaluronan and hyaluronan-aggrecan complexes," Biomacromolecules, vol. 12, no. 10 , pp. $3432-3443,2011$.

[106] R. M. A. Domingues, M. Silva, P. Gershovich et al., "Development of injectable hyaluronic acid/cellulose nanocrystals bionanocomposite hydrogels for tissue engineering applications," Bioconjugate Chemistry, vol. 26, no. 8, pp. 1571-1581, 2015.

[107] J. E. Frith, D. J. Menzies, A. R. Cameron et al., "Effects of bound versus soluble pentosan polysulphate in PEG/HAbased hydrogels tailored for intervertebral disc regeneration," Biomaterials, vol. 35, no. 4, pp. 1150-1162, 2014.

[108] A. Gilarska, J. Lewandowska-Łańcucka, W. Horak, and M. Nowakowska, "Collagen/chitosan/hyaluronic acid-based injectable hydrogels for tissue engineering applications-design, physicochemical and biological characterization," Colloids and Surfaces B: Biointerfaces, vol. 170, pp. 152-162, 2018.

[109] G. Bhakta, B. Rai, Z. X. H. Lim et al., "Hyaluronic acid-based hydrogels functionalized with heparin that support controlled release of bioactive BMP-2," Biomaterials, vol. 33, no. 26, pp. 6113-6122, 2012.

[110] J. R. Choi, K. W. Yong, J. Y. Choi, and A. C. Cowie, "Recent advances in photo-crosslinkable hydrogels for biomedical applications," Biotechniques, vol. 66, no. 1, pp. 40-53, 2019.

[111] C. B. Highley, G. D. Prestwich, and J. A. Burdick, "Recent advances in hyaluronic acid hydrogels for biomedical 
applications," Current Opinion in Biotechnology, vol. 40, pp. 35-40, 2016.

[112] N. E. Fedorovich, J. Alblas, J. R. de Wijn, W. E. Hennink, A. J. Verbout, and W. J. A. Dhert, "Hydrogels as extracellular matrices for skeletal tissue engineering: state-of-the-art and novel application in organ printing," Tissue Engineering, vol. 13, no. 8, pp. 1905-1925, 2007.

[113] W. Zhang and S. Chen, "Femtosecond laser nanofabrication of hydrogel biomaterial," MRS Bulletin, vol. 36, no. 12, pp. 1028-1033, 2011.

[114] A. Skardal, J. Zhang, L. McCoard, S. Oottamasathien, and G. D. Prestwich, "Dynamically crosslinked gold nanoparticle-hyaluronan hydrogels," Advanced Materials, vol. 22, no. 42, pp. 4736-4740, 2010.

[115] M. Hemshekhar, R. M. Thushara, S. Chandranayaka, L. S. Sherman, K. Kemparaju, and K. S. Girish, "Emerging roles of hyaluronic acid bioscaffolds in tissue engineering and regenerative medicine," International Journal of Biological Macromolecules, vol. 86, pp. 917-928, 2016. 


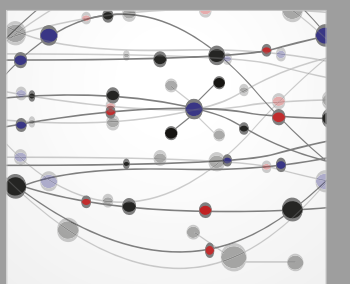

The Scientific World Journal
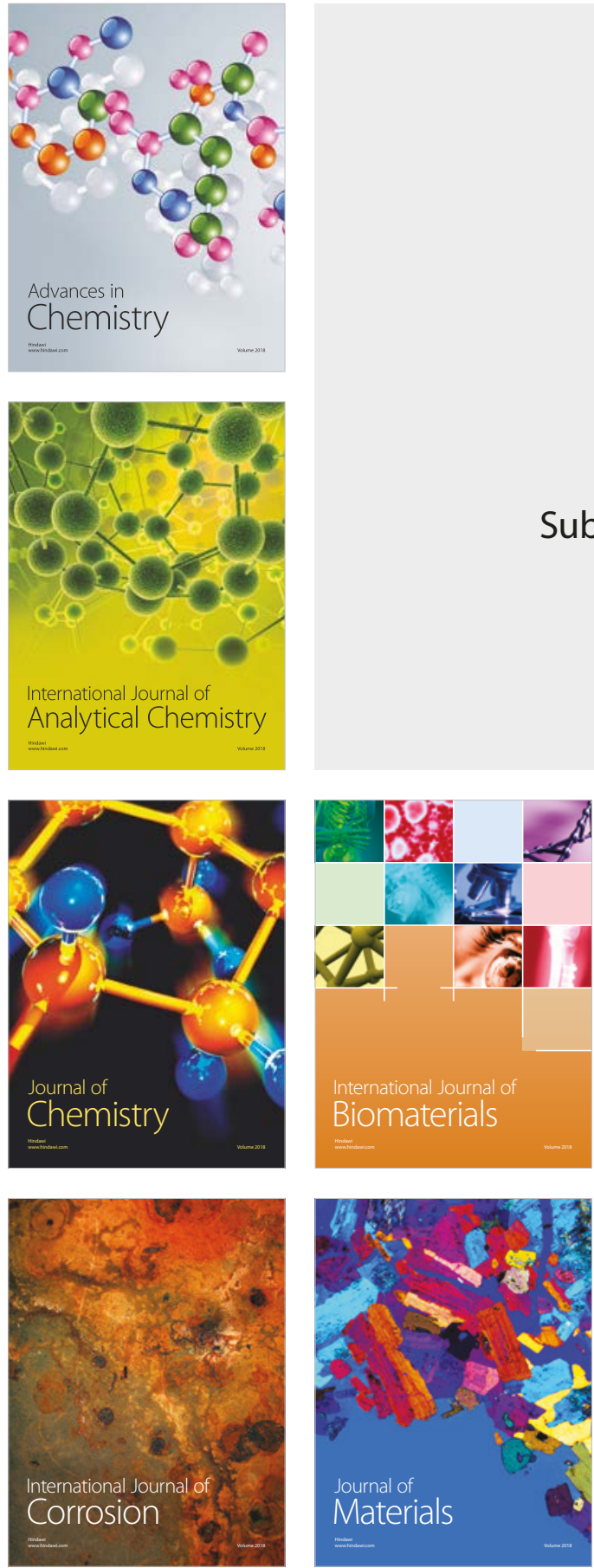

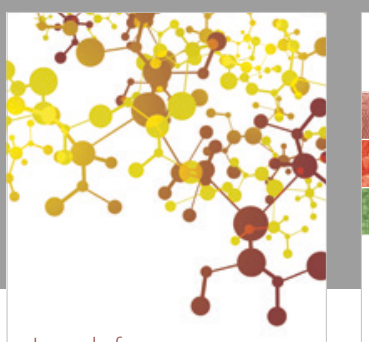

Journal of

Applied Chemistry
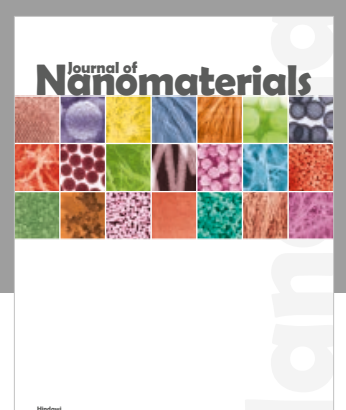

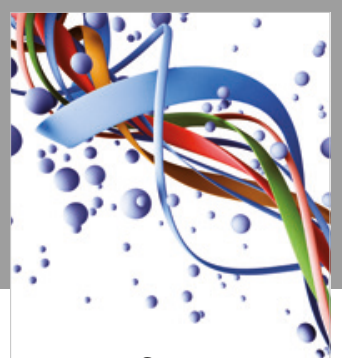

Scientifica

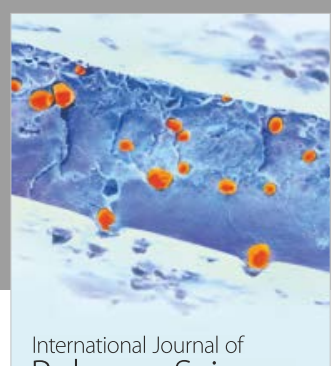

Polymer Science

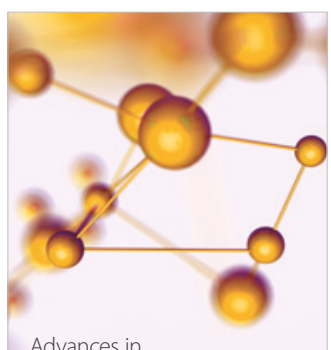

Physical Chemistry
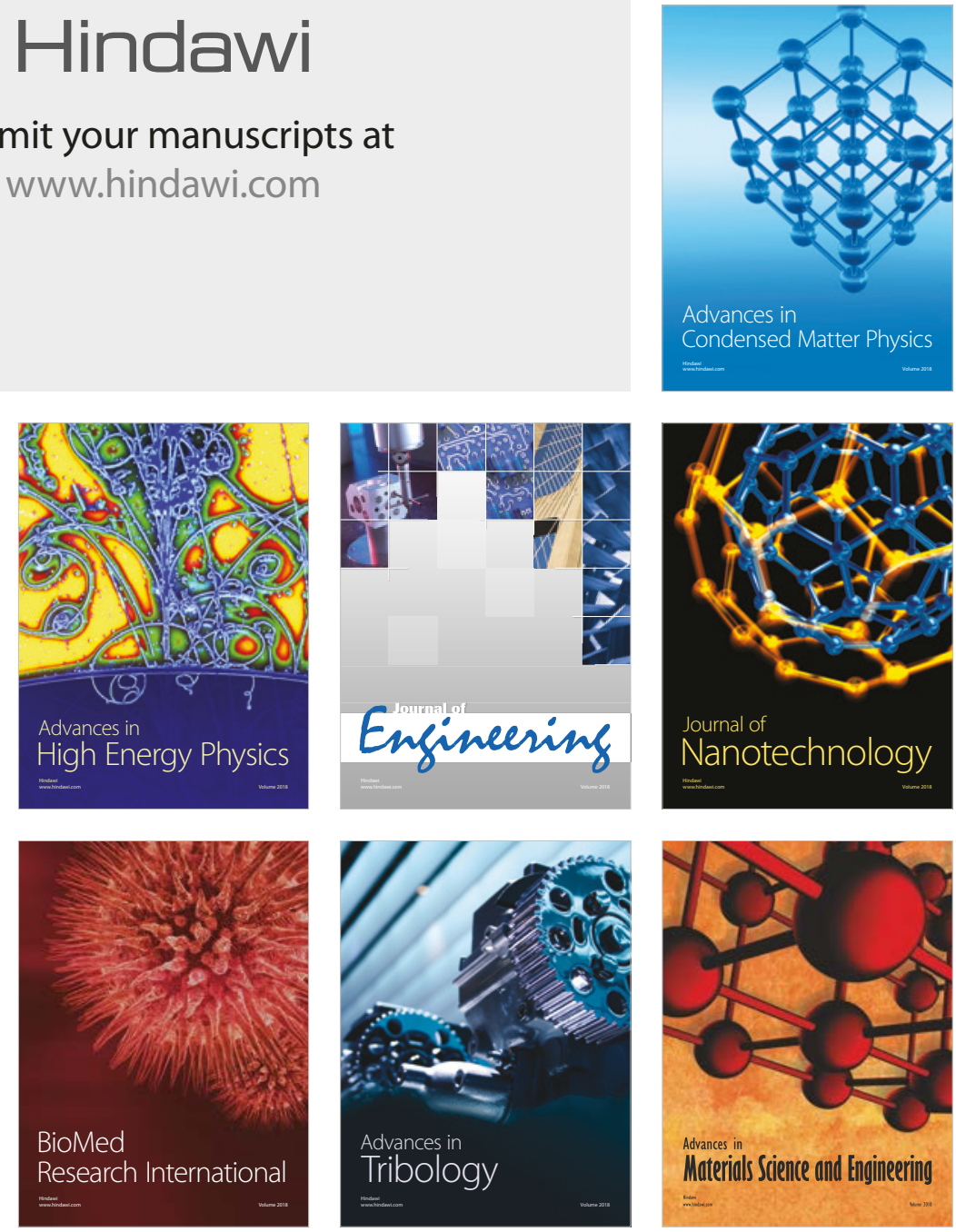